\title{
THE CLASSIFICATION OF COMPLETE MINIMAL SURFACES WITH TOTAL CURVATURE GREATER THAN $-12 \pi$
}

\author{
FRANCISCO J. LOPEZ
}

\begin{abstract}
We classify complete orientable minimal surfaces with finite total curvature $-8 \pi$.
\end{abstract}

\section{INTRODUCTION}

The classification of complete minimal surfaces with finite total curvature in

$\mathbf{R}^{3}$ has been an important problem in the classical differential geometry.

Some basic properties of these surfaces were studied by R. Osserman (see $[8,9])$, who showed the first nontrivial result about this subject.

Concretely, he characterized the catenoid and Enneper surface as the unique complete orientable minimal surfaces of total curvature $-4 \pi$.

However until recent years no more relevant results have been obtained.

W. H. Meeks [6] gave the classification of nonorientable complete minimal surfaces with total curvature greater than $-8 \pi$.

This paper is concerned with the total classification of orientable complete minimal surfaces with total curvature $-8 \pi$.

Chen and Gackstatter [1] discovered the first example of a complete minimal surface properly of genus 1 (see Theorem 1). The picture of Chen-Gackstatter surface is obtained by joining a handle on Enneper's surface. This genus one minimal surface has total curvature $-8 \pi$, and no other examples of such surfaces were found.

So, it is expected that no other genus one orientable minimal surface of total curvature $-8 \pi$ does exist.

In this paper we give a proof of this fact. More precisely, we prove that

"Chen-Gackstatter surface is the only genus one orientable complete minimal surface with finite total curvature $-8 \pi$."

Of course, it is not difficult to find genus zero minimal surfaces with total curvature $-8 \pi$.

A geometrically interesting example, described by Jorge and Meeks [5], is the trinoid.

This surface has three embedded catenoid ends. Moreover its normal vectors at these ends are placed symmetrically in an equator of $\mathbf{S}^{2}$.

Received by the editors April 27, 1990 and, in revised form, July 20, 1990.

1980 Mathematics Subject Classification (1985 Revision). Primary 53A10.

Research partially supported by DGICYT Grant PS87-0115-C03-02.

(C) 1992 American Mathematical Society $0002-9947 / 92 \$ 1.00+\$ .25$ per page 
To find all the genus zero orientable complete minimal surfaces of total curvature $-8 \pi$ is merely an elementary algebraic problem (see $\S 3$ ).

These facts together with the above Osserman result complete our classification.

Finally, in $\S 5$, we prove a modified version of the Osserman-Mo Theorem [7] for complete minimal surfaces.

It is interesting to notice that recently C. Costa [2] has obtained the classification of genus one embedded complete minimal surfaces in $\mathbf{R}^{3}$ of finite total curvature $-12 \pi$. All these surfaces lie in a smooth one-parameter family of tori punctured in three points, which correspond to three catenoid ends, except Costa's surface which has two catenoid ends and a planar end. This family was described by Hoffman and Meeks (see [3 and 4]).

On the other hand, Chen and Gackstatter [1] constructed an example of a genus two orientable minimal surface punctured in a point with total curvature $-12 \pi$.

So, to exhibit the total classification of such surfaces could be an interesting open problem.

The editor sent me two Ph.D. theses by E. L. Barbanel [12] and Y. Fang [13].

The first one develops the classification and study of genus zero complete minimal surfaces of total curvature $-8 \pi$, and the second one includes a partial version of this classification in the genus one case.

\section{Preliminaries}

In this section we expose some basic results about orientable complete minimal surfaces of finite total curvature. For more details, see [9, Chapter 9] and [8].

Let $x: M \rightarrow \mathbf{R}^{3}$ be an orientable complete minimal surface of finite total curvature in the Euclidean space $\mathbf{R}^{3}$.

Denote by $g, \omega$ the meromorphic function and the holomorphic 1-form determined by the Weierstrass representation of $x$ [9].

It is well known that, modulo natural identifications, $g$ is the Gauss map of $M$. Moreover, $g \omega$ and $g^{2} \omega$ are holomorphic 1 -forms and

$$
x=\operatorname{Re} \int\left(\phi_{1}, \phi_{2}, \phi_{3}\right)
$$

where $\phi_{1}=\left(1-g^{2}\right) \omega / 2, \phi_{2}=i\left(1+g^{2}\right) w / 2$ and $\phi_{3}=g \omega$.

Osserman proved (see [9]) that $M$ is conformally equivalent to a compact Riemann surface $\bar{M}$ punctured in a finite set of points $\left\{P_{1}, \ldots, P_{k}\right\}: M=$ $\bar{M}-\left\{P_{1}, \ldots, P_{k}\right\}$.

The points $P_{1}, \ldots, P_{k}$, correspond to the ends of $M$, and $g, \omega$ extend meromorphically to $\bar{M}$. Then $\phi_{i}, i=1,2,3$, have poles of order $\operatorname{Ord}_{p_{j}} \phi_{i}$ at $P_{j}, j=1, \ldots, k$.

If we put

$$
\begin{gathered}
n=\operatorname{Degree}(g), \quad \gamma=\operatorname{Genus}(M), \\
I_{j}+1=\max \left\{\operatorname{Ord}_{P_{j}} \phi_{i}, i=1,2,3\right\}, \quad j=1, \ldots, k,
\end{gathered}
$$


Jorge-Meeks formula gives (see [5])

$$
2 n=2 \gamma-2+\sum_{j=1}^{k}\left(I_{j}+1\right) .
$$

In the following, we will assume that $n=2$, i.e., $M$ has total curvature $-8 \pi$.

Using (2) and taking into account that $I_{j} \geq 1, j=1, \ldots, k$, we deduce $\gamma \leq 1$ (observe that the case $\gamma=2, k=1, I_{1}=1$ is impossible).

We describe the distinct possibilities in the following table:

TABLE 1

\begin{tabular}{|l|l|c|c|}
\hline$n=2$ & $k=1$ & $k=2$ & $k=3$ \\
\hline$\gamma=0$ & $I_{1}=5$ & $\begin{array}{c}I_{1}=I_{2}=2 \\
I_{1}=1, I_{2}=3\end{array}$ & $I_{1}=I_{2}=I_{3}=1$ \\
\hline$\gamma=1$ & $I_{1}=3$ & \\
\hline
\end{tabular}

Schoen has characterized the catenoid as the unique surface with $k=2$ and $I_{1}=I_{2}=1$ (see [10]). From (2), the case $k=3, \gamma=1$ is impossible.

We will classify all the surfaces which correspond to the other possibilities in the above table.

\section{GENUS ONE MINIMAL SURFACES OF TOTAL CURVATURE $-8 \pi$}

Throughout this section, we assume $\gamma=1, n=2$ and therefore, $k=1$, $I_{1}=3$. We write $M=\bar{M}-\{P\}$, where $\bar{M}$ is a genus one compact Riemann surface and $P$ is the end of $M$.

It is clear that $b_{g}(P) \leq 1$, where $b_{g}(P)$ is the branch number of $g$ at $P$.

We will discuss separately the case when $b_{g}(P)=0$ and $b_{g}(P)=1$.

2.1. First case. $b_{g}(P)=0$. Consider the following initial value problems of linear differential equations in the complex domain:

$$
\begin{aligned}
& \left\{\begin{array}{l}
f_{i}^{\prime}(a)=\frac{-a}{2\left(1-a^{2}\right)} f_{i}(a)+\frac{3}{4\left(1-a^{2}\right)} g_{i}(a), \\
g_{i}^{\prime}(a)=\frac{1}{\left(1-a^{2}\right)} f_{i}(a)-\frac{3 a}{2\left(1-a^{2}\right)} g_{i}(a),
\end{array}\right. \\
& f_{1}(0)=\int_{-1}^{0} \sqrt{\frac{x}{x^{2}-1}} d x, \quad g_{1}(0)=\int_{-1}^{0} \sqrt{\frac{x^{2}-1}{x}} d x, \\
& f_{2}(0)=\int_{0}^{1} \sqrt{\frac{x}{x^{2}-1}} d x, \quad g_{2}(0)=\int_{0}^{1} \sqrt{\frac{x^{2}-1}{x}} d x,
\end{aligned}
$$

where we have fixed in each case the branch of $\sqrt{\left(x^{2}-1\right) / x}$ such that $f_{1}(0)$, $g_{1}(0)<0$ and $f_{2}(0)=i f_{1}(0), g_{2}(0)=-i g_{2}(0)$.

Take $a, b \in \mathbf{C}$, and define $[a, b]=\{t a+(1-t) b \mid t \in[0,1] \subset \mathbf{R}\}$. We will write $\int_{a}^{b} h(x) d x$ instead of $\int_{[a, b]} h(x) d x$, for each function $h$ defined on $[a, b]$. 
Lemma 1. The initial value problems $\left(\mathrm{I}_{i}\right), i=1,2$, have well-defined solutions $f_{i}, g_{i}, i=1,2$, on the domain $\mathbf{C}-\{y \in \mathbf{R}|| y \mid>1\}$.

In fact, the solutions of $\left(\mathbf{I}_{i}\right), i=1,2$, are the functions

$$
\begin{array}{ll}
f_{1}(a)=\int_{-1}^{a} \sqrt{\frac{x-a}{x^{2}-1}} d x, & g_{1}(a)=\int_{-1}^{a} \sqrt{\frac{x^{2}-1}{x-a}} d x, \\
f_{2}(a)=\int_{a}^{1} \sqrt{\frac{x-a}{x^{2}-1}} d x, & g_{2}(a)=\int_{a}^{1} \sqrt{\frac{x^{2}-1}{x-a}} d x
\end{array}
$$

respectively, for a suitable single-valued branch of $\sqrt{\left(x^{2}-1\right) /(x-a)}, a \in \mathbf{C}-$ $\{y \in \mathbf{R} \| y \mid>1\}, x \in[-1, a] \cup[a, 1]$.

Moreover,

$$
\begin{gathered}
f_{2}(a)=i f_{1}(-a), \quad g_{2}(a)=-i g_{1}(-a), \\
f_{1}(\bar{a})=\bar{f}_{1}(a) \quad \text { and } \quad g_{1}(\bar{a})=\bar{g}_{1}(a) .
\end{gathered}
$$

Proof. The function $F:[0,1] \times(\mathbf{C}-\{y \in \mathbf{R} \mid y \geq 1\}) \rightarrow \mathbf{C}$ defined by

$$
F(t, a)=(a+1) t-2
$$

has rank $\mathbf{C}-\{y \in \mathbf{R} \mid y>0\}$.

Then, $\sqrt{F}$ has a single-valued branch on $[0,1] \times(\mathbf{C}-\{y \in \mathbf{R} \mid y>1\})$, and so $\sqrt{(t-1) /\left((a+1) t^{2}-2 t\right)}$.

Since

$$
\begin{aligned}
& f_{1}(a)=(a+1) \int_{0}^{1} \sqrt{\frac{t-1}{(a+1) t^{2}-2 t}} d t, \\
& g_{1}(a)=(a+1) \int_{0}^{1} \sqrt{\frac{(a+1) t^{2}-2 t}{t-1}} d t,
\end{aligned}
$$

we deduce that $f_{1}, g_{1}$ are well defined and holomorphic on $\mathbf{C}-\{y \in \mathbf{R} \mid y \geq 1\}$, and $f_{1}(0), g_{1}(0)<0$ for a suitable election of the above branch.

Analogously, $f_{2}, g_{2}$ are holomorphic functions on $\mathbf{C}-\{y \in \mathbb{R} / y \leq-1\}$, and can be chosen such that $f_{2}(0)=i f_{1}(0), g_{2}(0)=-i g_{1}(0)$.

On the other hand

$$
f_{1}^{\prime}(a)=\int_{0}^{1} \sqrt{\frac{t-1}{(a+1) t^{2}-2 t}} d t-\frac{a+1}{2} \int_{0}^{1} \sqrt{\frac{(t-1) t}{((a+1) t-2)^{3}}} d t
$$

and integrating by parts

$$
f_{1}^{\prime}(a)=\frac{a}{2(a-1)} \int_{0}^{1} \sqrt{\frac{t-1}{(a+1) t^{2}-2 t}} d t-\frac{3}{4(a-1)} \int_{0}^{1} \sqrt{\frac{(a+1) t^{2}-2 t}{t-1}} d t .
$$


Analogously

$$
g_{1}^{\prime}(a)=\frac{-1}{a-1} \int_{0}^{1} \sqrt{\frac{t-1}{(a+1) t^{2}-2 t}} d t+\frac{3 a}{2(a-1)} \int_{0}^{1} \sqrt{\frac{(a+1) t^{2}-2 t}{t-1}} d t .
$$

Hence, $f_{1}, g_{1}$ verify $\left(\mathrm{I}_{1}\right)$.

In a similar way, $f_{2}, g_{2}$ verify $\left(\mathbf{I}_{2}\right)$.

At last, observe that $i f_{1}(-a),-i g_{1}(-a)$ satisfy the initial value problem $\left(\mathbf{I}_{2}\right)$, and by the uniqueness of solutions, $i f_{1}(-a)=f_{2}(a),-i g_{1}(-a)=g_{2}(a)$.

Taking into account that $g_{1}(a), f_{1}(a) \in \mathbf{R}$ if $a \in[-1,1]$, and the analyticity of the solutions, (4) holds and the proof is complete. Q.E.D.

Remark 1. Note that $f_{i}, g_{i}, i=1,2$, are also the solutions of the following initial value problems

$$
f_{i}^{\prime \prime}(a)=\frac{1}{4\left(1-a^{2}\right)} f_{i}(a), \quad g_{i}^{\prime \prime}(a)=\frac{-3}{4\left(1-a^{2}\right)} g_{i}(a), \quad i=1,2,
$$

(6) $f_{1}(0)=\int_{-1}^{0} \sqrt{\frac{x}{x^{2}-1}} d x, \quad g_{1}(0)=\int_{-1}^{0} \sqrt{\frac{x^{2}-1}{x}} d x$,

$$
\begin{array}{ll}
f_{2}(0)=i f_{1}(0), & g_{2}(0)=-i g_{1}(0), \\
f_{i}^{\prime}(0)=\frac{3}{4} g_{i}(0), & g_{i}^{\prime}(0)=f_{i}(0), \quad i=1,2 .
\end{array}
$$

Remark 2. The functions $f_{1}, g_{1}$ are defined and holomorphic on $\mathbf{C}-\{y \in \mathbf{R} \mid$ $y \geq 1\}$ and have both a simple zero at -1 . Analogously, $f_{2}, g_{2}$ are defined and holomorphic on $\mathbf{C}-\{y \in \mathbf{R} \mid y \leq-1\}$ and have both a simple zero at 1 .

Consider the Riemann sphere $\mathbf{C} \cup\{\infty\}$, and draw a straight line $l$ from 1 to -1 passing by $\infty$ along the real axis. Then cut and open along $l$.

The closure $\Omega$ of the resulting domain has two copies of the line $l$. Call one of them $l_{1}$ and the other one $l_{2}$ (see Figure 1).

We will put $a_{1}, a_{2}$, the two points corresponding to $a \in l$, in $l_{1}$ and $l_{2}$ respectively.

Observe that $\infty_{1} \neq \infty_{2}$ and $1_{1}=1_{2},-1_{1}=-1_{2}$.

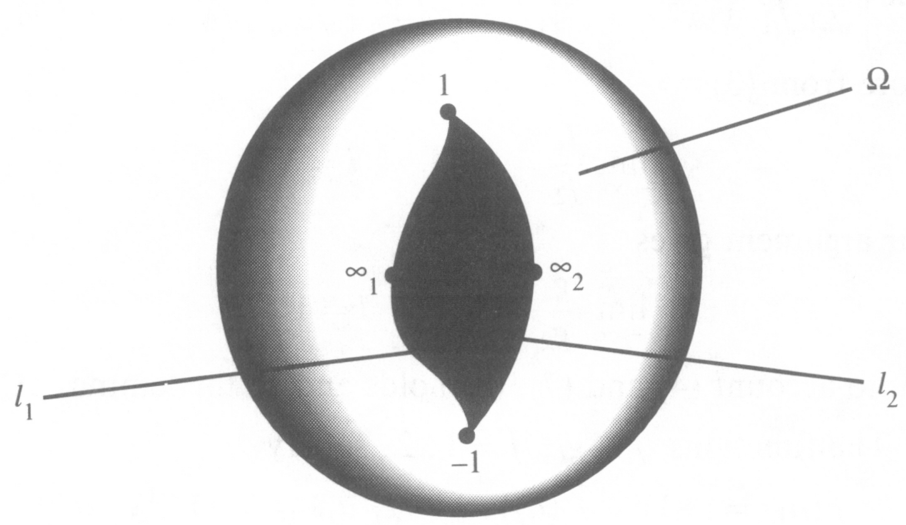

FIGURE 1 
Lemma 2. The functions $f_{i}, g_{i}, i=1,2$, extend continuously to $\Omega-\left\{\infty_{1}, \infty_{2}\right\}$, satisfying

(7)

$$
\begin{gathered}
f_{1}(a), g_{1}(a) \neq 0, \quad a \neq \infty_{1}, \infty_{2},-1, \quad f_{1}(1)=-2 \sqrt{2}, \quad g_{1}(1)=-\frac{4 \sqrt{2}}{3}, \\
f_{1}^{\prime}(-1)=\int_{0}^{1} \sqrt{\frac{1-t}{2 t}} d t<0, \quad g_{1}^{\prime}(-1)=2 f_{1}^{\prime}(-1), \\
f_{2}(a), g_{2}(a) \neq 0, \quad a \neq \infty_{1}, \infty_{2}, 1, \quad f_{2}(-1)=i f_{1}(1), \quad g_{2}(-1)=-i g_{1}(1), \\
f_{2}^{\prime}(1)=-i f_{1}^{\prime}(-1), \quad g_{2}^{\prime}(1)=i g_{1}^{\prime}(-1)
\end{gathered}
$$

and

$$
\begin{gathered}
\lim _{a \rightarrow \infty}\left|\frac{\sqrt{a}}{f_{i}}\right|=0, \quad \lim _{a \rightarrow \infty j}\left|\frac{a \sqrt{a}}{g_{i}}\right| \neq 0, \infty, \quad \lim _{a \rightarrow \infty_{j}}\left|\frac{a^{\varepsilon} \sqrt{a}}{f_{i}}\right|=\infty, \quad \varepsilon>0, \\
\lim _{a \rightarrow 1} \frac{f_{1} g_{2}}{f_{2} g_{1}}=-3, \quad \lim _{a \rightarrow-1} \frac{f_{1} g_{2}}{f_{2} g_{1}}=-\frac{1}{3} \quad \lim _{a \rightarrow \infty} \frac{f_{1} g_{2}}{f_{2} g_{1}}=1
\end{gathered}
$$

where $i=1,2$ and $j=1,2$.

Proof. Using (3), it is straightforward to check that $f_{i}, g_{i}, i=1,2$, extend continuously to $\Omega-\left\{\infty_{1}, \infty_{2}, 1,-1\right\}$ and $f_{1}(a), g_{1}(a) \neq 0$ if $\operatorname{Im}(a)=0$, $a \neq \infty_{1}, \infty_{2},-1$.

Notice that if $\operatorname{Im}(a) \neq 0, \operatorname{Im} \sqrt{2-(a+1)} t \neq 0$, where $t \in] 0,1]$. Thus, from (5), $f_{1}(a) \neq 0$, and in the same way, $g_{1}(a) \neq 0$. So, from (4), we have $f_{2}(a), g_{2}(a) \neq 0, a \neq \infty_{1}, \infty_{2}, 1$.

Using now (4), (5) again, it is not hard to prove (7). On the other hand, (5) yields

$$
\begin{gathered}
\lim _{a \rightarrow \infty}\left|\frac{\sqrt{a}}{f_{1}}\right|=\left|\int_{0}^{1} \sqrt{\frac{1-t}{t^{2}}} d t\right|^{-1}=0, \quad \lim _{a \rightarrow \infty_{i}}\left|\frac{a \sqrt{a}}{g_{1}}\right|=\left|\int_{0}^{1} \sqrt{\frac{t^{2}}{1-t}} d t\right| \neq 0, \\
\lim _{a \rightarrow \infty}\left|\frac{a^{\varepsilon} \sqrt{a}}{f_{1}}\right|=\infty
\end{gathered}
$$

where $i=1,2$, and the same holds for $f_{2}, g_{2}$ (see (4)).

Moreover

$$
\lim _{a \rightarrow \infty}\left|\frac{1}{\sqrt{a}} \int_{0}^{1} \sqrt{\frac{x-a}{x^{2}-1}} d x\right|, \quad \lim _{a \rightarrow \infty}\left|\frac{1}{\sqrt{a}} \int_{-1}^{0} \sqrt{\frac{x-a}{x^{2}-1}} d x\right| \neq 0
$$

and therefore from (3),

$$
\lim _{a \rightarrow \infty} \frac{f_{1}}{f_{2}}=-1, \quad i=1,2 .
$$

A similar argument gives

$$
\lim _{a \rightarrow \infty} \frac{g_{1}}{g_{2}}=1, \quad i=1,2 .
$$

Taking into account (4) and (7), (8) holds and so the lemma. Q.E.D.

Remark 3. The functions $f_{i}, g_{i}, i=1,2$, satisfy

$$
f_{i}\left(a_{1}\right)=(-1)^{i-1} \bar{f}_{i}\left(a_{2}\right), \quad g_{i}\left(a_{1}\right)=(-1)^{i-1} \bar{g}_{i}\left(a_{2}\right)
$$

where $a_{i} \in l_{i}, i=1,2, a \neq \infty$. 
Remark 4. Observe that $\left.\left.f_{1}(a), g_{1}(a) \in \mathbf{R}, a \in\right]-\infty, 1\right], f_{2}(a) / i, g_{2}(a) / i \in \mathbf{R}$, $a \in[-1,+\infty[$. Moreover

$$
\begin{gathered}
\left.\left.f_{1}(a), g_{1}(a)>0, \quad a \in\right]-\infty,-1\left[, \quad f_{1}(a), g_{1}(a)<0, \quad a \in\right]-1,1\right] \\
\left.\frac{1}{i} f_{2}(a), \quad-\frac{1}{i} g_{2}(a)<0, \quad a \in\right]-1,1[, \\
\operatorname{Re}\left(f_{1}\left(a_{i}\right)\right), \quad \operatorname{Re}\left(g_{1}\left(a_{i}\right)\right)<0, \\
(-1)^{i} \operatorname{Im}\left(f_{1}\left(a_{i}\right)\right)=\frac{1}{i} f_{2}\left(a_{1}\right)=\frac{1}{i} f_{2}\left(a_{2}\right)>0, \\
(-1)^{i} \operatorname{Im}\left(g_{1}\left(a_{i}\right)\right)=\frac{1}{i} g_{2}\left(a_{1}\right)=\frac{1}{i} g_{2}\left(a_{2}\right)<0,
\end{gathered}
$$

where $a_{i} \in l_{i}-\left\{\infty_{i}\right\}, a_{i}>1, i=1,2$.

Remark 5. The functions $f_{i}, g_{i}$ satisfy $\left(\mathrm{I}_{1}\right), i=1,2$, on

$$
\left(l_{1} \cup l_{2}\right)-\left\{\infty_{1}, \infty_{2}, 1,-1\right\} .
$$

Lemma 3. If $a \in \Omega-\left\{\infty_{1}, \infty_{2}\right\}$,

$$
\left(f_{1} g_{2}-f_{2} g_{1}\right)(a)=\frac{4 \pi i}{3}\left(1-a^{2}\right) \text {. }
$$

Proof. Consider the genus one compact Riemann surface

$$
\bar{M}_{a}=\left\{(z, w) \in(\mathbf{C} \cup\{\infty\})^{2} \mid w^{2}=(z-a)\left(z^{2}-1\right)\right\} \text {, where } a \in \mathbf{C}-\{1,-1\} .
$$

We can construct a "concrete" representation of $\bar{M}_{a}$ as a two-sheeted covering of the sphere $\mathbf{C} \cup\{\infty\}$.

Picture two copies of the sphere, and label these two copies sheet I and sheet II. On each sheet, cut along two smooth curves joining -1 to " $a$ " and 1 to $\infty$, in such way that these cuts do not intersect.

Each "cut" has two banks; an N-bank and an S-bank.

Joining every S-bank on sheet I to the N-bank of the corresponding "cut" on sheet II, and then joining the corresponding S-bank on sheet II to the N-bank of the corresponding "cut" on sheet I, we have the desired representation of $\bar{M}_{a}$ (see Figure 2).

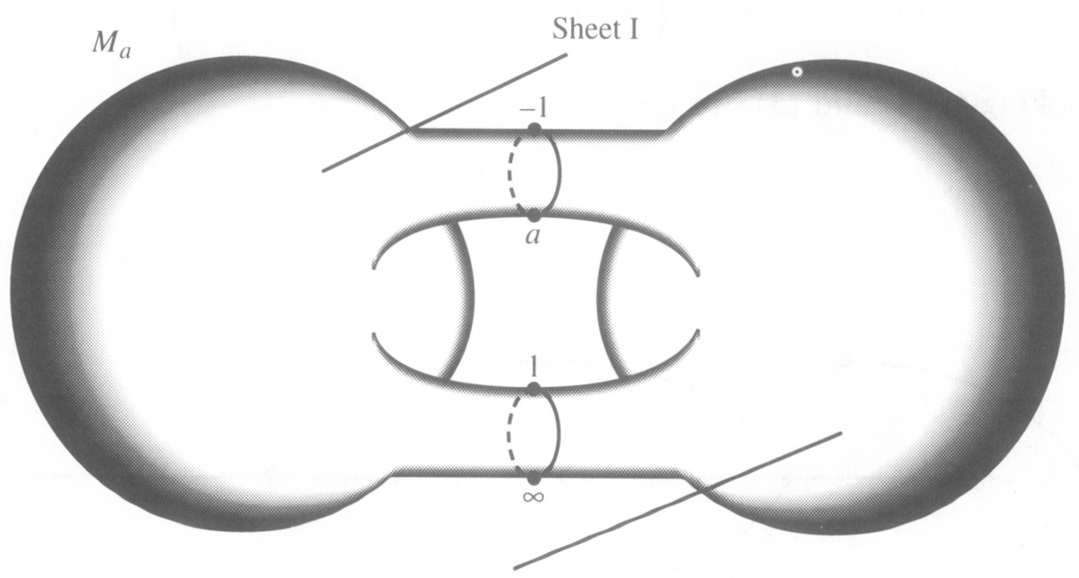

Sheet II

FIGURE 2 
We can construct a canonical homology basis for $\bar{M}_{a}$ drawing simple smooth curves $\gamma_{i}, i=1,2 ; \gamma_{1}$ is given winding once around the "cut" from -1 to " $a$ " in one sheet of $\bar{M}_{a}$, and $\gamma_{2}$ starting from a point on "cut" from -1 to " $a$ " going on the first sheet to a point on "cut" from 1 to $\infty$, and returning on the second sheet (indicated in Figure 3 below by dotted lines) to the original point.

The orientation of $\gamma_{1}, \gamma_{2}$ is illustrated in Figure 3.

Define now the meromorphic 1-forms:

$$
\tau_{1}^{a}=\frac{z-a}{w} d z, \quad \tau_{2}^{a}=\frac{w}{z-a} d z
$$

and observe that

$$
2 f_{i}(a)=\int_{\gamma_{i}} \tau_{1}^{a}, \quad 2 g_{i}(a)=\int_{\gamma_{i}} \tau_{2}^{a}, \quad i=1,2 .
$$

Using standard bilinear relations (see [11]), we conclude the proof. Q.E.D.

Lemma 4. If $a_{i} \in l_{i}-\left\{\infty_{i}\right\}, i=1,2$, then

$$
\left|f_{1} g_{2} / f_{2} g_{1}\right|\left(a_{i}\right) \neq 1 \text {. }
$$

Proof. Remark 3 involves $\left|f_{1} g_{2} / f_{2} g_{1}\right|\left(a_{1}\right)=\left|f_{1} g_{2} / f_{2} g_{1}\right|\left(a_{2}\right)$. So we need only to prove the lemma for $a_{1} \in l_{1}-\{\infty\}$.

Suppose $a_{1}>1$.

From Remark 4 and Lemma 3

$$
\left|\frac{f_{1} g_{2}}{f_{2} g_{1}}\right|\left(a_{1}\right)=1 \Leftrightarrow\left(\frac{f_{2}}{\operatorname{Re}\left(f_{1}\right)}+\frac{g_{2}}{\operatorname{Re}\left(g_{1}\right)}\right)\left(a_{1}\right)=0 .
$$

Let $t:] 1,+\infty[\rightarrow \mathbf{R}$ the function defined by

$$
t\left(a_{1}\right)=\left(2 \operatorname{Re}\left(f_{1}\right)-\sqrt{3} \operatorname{Re}\left(g_{1}\right)\right)\left(a_{1}\right) .
$$

Remarks 4, 5 and 1 yields

$$
\begin{gathered}
t^{\prime}\left(a_{1}\right)=\frac{1}{a_{1}^{2}-1}\left(\left(a_{1}+\sqrt{3}\right) \operatorname{Re}\left(f_{1}\right)\left(a_{1}\right)-\frac{3}{2}\left(1+\sqrt{3} a_{1}\right) \operatorname{Re}\left(g_{1}\right)\left(a_{1}\right)\right), \\
t^{\prime \prime}\left(a_{1}\right)=\frac{1}{2\left(1-a_{1}^{2}\right)}\left(\operatorname{Re}\left(f_{1}\right)\left(a_{1}\right)+\frac{3 \sqrt{3}}{2} \operatorname{Re}\left(g_{1}\right)\left(a_{1}\right)\right)>0 .
\end{gathered}
$$

Looking at (8) and (3), we have

$$
\lim _{a_{1} \rightarrow \infty} t^{\prime}\left(a_{1}\right)=0
$$

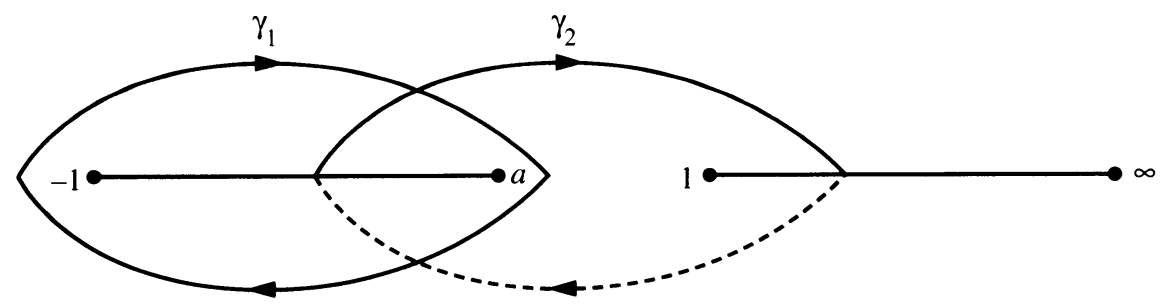

Figure 3 
and therefore

$$
\left.t^{\prime}\left(a_{1}\right)<0, \quad \text { for each } a_{1} \in\right] 1,+\infty[.
$$

But from (7), $t(1)<0$ and then

$$
t\left(a_{1}\right)<0, \quad \text { for each } a_{1} \in[1,+\infty[.
$$

If $\left|f_{1} g_{2} / f_{2} g_{1}\right|\left(a_{1}^{\prime}\right)=1, a_{1}^{\prime}>1$, from (9) and Remark 2,

$$
\left(\frac{f_{2}}{\operatorname{Re}\left(f_{1}\right)}+\frac{g_{2}}{\operatorname{Re}\left(g_{1}\right)}\right)\left(a_{1}^{\prime}\right)=\left(\frac{f_{2}}{\operatorname{Re}\left(f_{1}\right)}+\frac{g_{2}}{\operatorname{Re}\left(g_{1}\right)}\right)(1)=0
$$

and then there exists $b \in] 1, a_{1}^{\prime}[$ such that

$$
\left(\frac{f_{2}}{\operatorname{Re}\left(f_{1}\right)}+\frac{g_{2}}{\operatorname{Re}\left(g_{1}\right)}\right)^{\prime}(b)=0 .
$$

Taking into account Remarks 5, 4 and Lemma 3: $t(b)=0$. So, from (10), we get a contradiction and thus

$$
\left.\left|f_{1} g_{2} / f_{2} g_{1}\right|\left(a_{1}\right) \neq 1, \quad \text { for each } a_{1} \in\right] 1,+\infty[\text {. }
$$

Using (4) and (7), the same holds for $\left.a_{1} \in\right]-\infty,-1\left[\right.$ and $a_{1}=1,-1$. Q.E.D.

Remark 6. Observe that from the proof of Lemma 4 we can deduce

$$
\left.\frac{1}{i}\left(\operatorname{Re}\left(f_{1}\right) g_{2}+\operatorname{Re}\left(g_{1}\right) f_{2}\right)\left(a_{1}\right)>0 \text { if } a_{1} \in\right] 1,+\infty_{1}\left[\subset l_{1}\right. \text {. }
$$

\section{Lemma 5.}

$$
\left(f_{1} \bar{g}_{2} / f_{2} \bar{g}_{1}\right)(a) \neq 1, \quad \text { for each } a \in i \mathbf{R}-\{0\} .
$$

Proof. Write $t_{i}=\operatorname{Re}\left(f_{i}\right), s_{i}=\operatorname{Im}\left(f_{i}\right), u_{i}=\operatorname{Re}\left(g_{i}\right), v_{i}=\operatorname{Im}\left(g_{i}\right), i=1,2$.

Since $a \in i \mathbf{R}$, it is easy to check from (4) that

$$
\frac{f_{1} \bar{g}_{2}}{f_{2} \bar{g}_{1}}(a)=1 \Leftrightarrow \operatorname{Im}\left(f_{1} g_{1}\right)(a)=0 \Leftrightarrow\left(t_{1} v_{1}+s_{1} u_{1}\right)(a)=0
$$

In the following, we put $a=i y, y \in \mathbf{R}$ and write simply $t_{i}(y), s_{i}(y), u_{i}(y)$ and $v_{i}(y)$ instead of $t_{i}(i y), s_{i}(i y), u_{i}(i y)$ and $v_{i}(i y), i=1,2$. Also $t_{i}^{\prime}$, $t_{i}^{\prime \prime}$ will mean $d t_{i} / d y, d^{2} t_{i} / d y^{2}$ respectively, and the same for $s_{i}, u_{i}$ and $v_{i}$, $i=1,2$.

Using Lemma 1 and Remark 1, we deduce that the above functions verify the differential equations

$$
\begin{aligned}
& \left\{\begin{array} { l } 
{ t _ { i } ^ { \prime } = \frac { y } { 2 ( 1 + y ^ { 2 } ) } t _ { i } - \frac { 3 } { 4 ( 1 + y ^ { 2 } ) } v _ { i } , } \\
{ s _ { i } ^ { \prime } = \frac { y } { 2 ( 1 + y ^ { 2 } ) } s _ { i } + \frac { 3 } { 4 ( 1 + y ^ { 2 } ) } u _ { i } , }
\end{array} \quad \left\{\begin{array}{l}
u_{i}^{\prime}=\frac{-1}{1+y^{2}} s_{i}+\frac{3 y}{2\left(1+y^{2}\right)} u_{i}, \\
v_{i}^{\prime}=\frac{1}{1+y^{2}} t_{i}+\frac{3 y}{2\left(1+y^{2}\right)} v_{i},
\end{array}\right.\right. \\
& \left\{\begin{array} { l } 
{ t _ { i } ^ { \prime \prime } = \frac { - 1 } { 4 ( 1 + y ^ { 2 } ) } t _ { i } , } \\
{ s _ { i } ^ { \prime \prime } = \frac { - 1 } { 4 ( 1 + y ^ { 2 } ) } s _ { i } , }
\end{array} \quad \left\{\begin{array}{l}
u_{i}^{\prime \prime}=\frac{3}{4\left(1+y^{2}\right)} u_{i}, \\
v_{i}^{\prime \prime}=\frac{3}{4\left(1+y^{2}\right)} v_{i}
\end{array}\right.\right.
\end{aligned}
$$

where $i=1,2$. 
If $i=1$, the initial values are

$$
\begin{aligned}
& t_{1}(0)=f_{1}(0), \quad t_{1}^{\prime}(0)=0, \quad u_{1}(0)=g_{1}(0), \quad u_{1}^{\prime}(0)=0, \\
& s_{1}(0)=0, \quad s_{1}^{\prime}(0)=\frac{3}{4} g_{1}(0), \quad v_{1}(0)=0, \quad v_{1}^{\prime}(0)=f_{1}(0),
\end{aligned}
$$

and analogously if $i=2$.

Observe that (4) involves

$$
\frac{f_{1} \bar{g}_{2}}{f_{2} \bar{g}_{1}}(i y)=1 \Leftrightarrow \frac{f_{1} \bar{g}_{2}}{f_{2} \bar{g}_{1}}(-i y)=1 .
$$

Then from (11), it is sufficient to prove that

$$
\left(t_{1} v_{1}+s_{1} u_{1}\right)(y) \neq 0, \quad \text { for each } y>0 .
$$

First, we will show that

$$
u_{1}(y), v_{1}(y), s_{1}(y)<0, \quad \forall y>0,
$$

$\exists y_{0}>0$ such that $t_{1}\left(y_{0}\right)=0, \quad t_{1}(y)<0$ if $0<y<y_{0}$,

$$
\begin{aligned}
t_{1}(y) & >0 \text { if } y_{0}<y<+\infty, \\
\lim _{y \rightarrow+\infty} \frac{s_{1}}{t_{1}} & =-1, \quad \lim _{y \rightarrow+\infty} \frac{v_{1}}{u_{1}}=1 .
\end{aligned}
$$

If $u_{1}$ vanishes at some point $y^{\prime}>0$, take $y_{1}>0$ the first point such that $u_{1}\left(y_{1}\right)=0$. Since $g_{1}(0)<0,(12)$ and (13) yield

$$
\left.\left.u_{1}^{\prime \prime}(y), u_{1}^{\prime}(y), u_{1}(y)<0, \quad y \in\right] 0, y_{1}\right],
$$

a contradiction.

In a similar way, $v_{1}(y)<0$, for each $y>0$.

Suppose $s_{1}$ vanishes at $y^{\prime}>0$, and take as before $y_{1}>0$ the first point such that $s_{1}\left(y_{1}\right)=0$.

Using that $u_{1}\left(y_{1}\right)<0$ and (12), we have $s_{1}^{\prime}\left(y_{1}\right)<0$, which contradicts (13).

We know that $v_{1}(y)<0, y>0$. So, from (12), if $t_{1}\left(y_{0}\right)=0, y_{0}>0$, then $t_{1}^{\prime}\left(y_{0}\right)>0$. Since $t_{1}(0)<0$ (see (13)), $t_{1}$ vanishes at most at one point $y_{0}>0$.

On the other hand, from (3),

$$
t_{1}(y)=\operatorname{Re} \int_{-1}^{0} \sqrt{\frac{x-i y}{x^{2}-1}} d x+\operatorname{Re}\left(i \int_{0}^{y} \sqrt{i \frac{y-r}{r^{2}+1}} d r\right)
$$

and therefore

$$
\lim _{y \rightarrow+\infty} t_{1}(y)=+\infty, \quad \lim _{y \rightarrow+\infty} \frac{s_{1}}{t_{1}}(y)=-1 .
$$

It is now easy to deduce that $t_{1}$ vanishes at only one point $y_{0}>0$.

By similar arguments

$$
\lim _{y \rightarrow+\infty} \frac{v_{1}}{u_{1}}(y)=1
$$

and (14) holds.

To finish the lemma, from (11) and (14) it is sufficient to show

$$
\left(\frac{s_{1}}{t_{1}}+\frac{v_{1}}{u_{1}}\right)(y) \neq 0, \quad \text { for each } y>0, \quad y \neq y_{0},
$$

and note that from $(14),\left(s_{1} u_{1}+v_{1} t_{1}\right)\left(y_{0}\right)=\left(s_{1} v_{1}\right)\left(y_{0}\right) \neq 0$. 
Suppose now

$$
\left(\frac{s_{1}}{t_{1}}+\frac{v_{1}}{u_{1}}\right)\left(y_{1}\right)=0, \quad y_{1}>0 .
$$

If $y_{1}<y_{0}$, since $\left(s_{1} / t_{1}+v_{1} / u_{1}\right)(0)=0$, we have

$$
\left.\left(\frac{s_{1}}{t_{1}}+\frac{v_{1}}{u_{1}}\right)^{\prime}\left(y_{2}\right)=0, \quad \text { where } y_{2} \in\right] 0, y_{1}[\text {. }
$$

From (12)

$$
\left(\frac{s_{1}}{t_{1}}+\frac{v_{1}}{u_{1}}\right)^{\prime}\left(y_{2}\right)=\left(\left(\frac{u_{1} t_{1}+v_{1} s_{1}}{1+y^{2}}\right)\left(\frac{3}{4 t_{1}^{2}}+\frac{1}{u_{1}^{2}}\right)\right)\left(y_{2}\right) .
$$

Thus $\left(u_{1} t_{1}+v_{1} s_{1}\right)\left(y_{2}\right)=0$, that is, $\operatorname{Re}\left(f_{1} \bar{g}_{1}\right)\left(i y_{2}\right)=0$.

But Lemma 3 and (4) give

$$
\operatorname{Re}\left(f_{1} \bar{g}_{1}\right)(-y)=-\frac{2 \pi}{3}\left(1+y^{2}\right) \neq 0, \quad \text { for each } y \in \mathbf{R}
$$

which gets a contradiction.

If $y_{0}<y_{1}$, observe that from (14)

$$
\lim _{y \rightarrow+\infty}\left(\frac{s_{1}}{t_{1}}+\frac{v_{1}}{u_{1}}\right)=0 .
$$

Reasoning as before, this case is also impossible. Q.E.D.

Remark 7. Note that

$$
\begin{array}{ll}
\left(u_{1} s_{1}+v_{1} t_{1}\right)(y)>0, & \text { for each } y>0, \\
\left(u_{1} s_{1}+v_{1} t_{1}\right)(y)<0, & \text { for each } y<0
\end{array}
$$

We can now state the main theorem of this section.

Theorem 1. Let $x: M \rightarrow \mathbf{R}^{3}$ be a orientable complete minimal surface of finite total curvature $-8 \pi$ and genus one.

Suppose that its Gauss map is regular at the unique end of $M$.

Then up to homothety and rigid motion, $x$ is given by

$$
x: \bar{M}-\{(\infty, \infty)\} \rightarrow \mathbf{R}^{3}, \quad x=\operatorname{Re} \int\left(\phi_{1}, \phi_{2}, \phi_{3}\right)
$$

where

$$
\begin{gathered}
\bar{M}=\left\{(z, w) \in(\mathbf{C} \cup\{\infty\})^{2} \mid w^{2}=z\left(z^{2}-1\right)\right\}, \\
\phi_{1}=\frac{1}{2}\left(\frac{z}{w}-A^{2} \frac{w}{z}\right) d z, \quad \phi_{2}=\frac{i}{2}\left(\frac{z}{w}+A^{2} \frac{w}{z}\right) d z, \\
\phi_{3}=A d z \quad \text { and } A^{2}=\frac{f_{1}}{g_{1}}(0) .
\end{gathered}
$$

Proof. We know that $M$ is conformally equivalent to $\bar{M}-\{P\}$, where $\bar{M}$ is a compact Riemann surface and $P$ is a point of $\bar{M}$.

If $g$ is the Gauss map of $M$, after a rotation in $\mathbf{R}^{3}$, we can suppose that $g(P)=\infty$. Since $b_{g}(P)=0$, there exist four points $P_{i}, i=1,2,3,4$, in $\bar{M}$ such that $b_{g}\left(P_{i}\right)=1, i=1,2,3,4$. We call $a_{i}=g\left(P_{i}\right), i=1,2,3,4$, and observe that $a_{i} \neq a_{j}, i \neq j$. 
Therefore $\bar{M}$ is conformally equivalent to

$$
\left\{\left(z_{1}, w_{1}\right) \in(\mathbf{C} \cup\{\infty\})^{2} \mid w_{1}^{2}=\left(z_{1}-a_{1}\right)\left(z_{1}-a_{2}\right)\left(z_{1}-a_{3}\right)\left(z_{1}-a_{4}\right)\right\}
$$

where $g=z_{1}$.

After a suitable change of parameter

$\bar{M}=\left\{\left(z_{2}, w_{2}\right) \in(\mathbf{C} \cup\{\infty\})^{2} \mid w_{2}^{2}=\left(z_{2}^{2}-1\right)\left(z_{2}-c_{1}\right)\left(z_{2}-d_{1}\right)\right\}, \quad g=A_{1} z_{2}+B_{1}$, where $c_{1}, d_{1}, A_{1}, B_{1} \in \mathbf{C}, c_{1} \neq d_{1}, c_{1}, d_{1} \neq 1,-1, A_{1} \neq 0$.

As $z_{2}(P)=g(P)=\infty$ and $b_{g}(P)=0$, there exists $Q \in \bar{M}$ such that $z_{2}(Q)=g(Q)=\infty$.

Without loss of generality, we can suppose that the meromorphic function on $\bar{M}: S$, defined by

$$
S\left(z_{2}, w_{2}\right)=w_{2}+z_{2}^{2}-\frac{c_{1}+d_{1}}{2} z_{2}+\left(\frac{c_{1} d_{1}-1}{2}-\frac{\left(c_{1}+d_{2}\right)^{2}}{8}\right)
$$

satisfy:

$$
S(P)=\infty, \quad b_{s}(P)=1, \quad S(Q)=0, \quad \operatorname{Degree}(S)=2 .
$$

From (1), (2), the holomorphic 1-form $\omega$ determined by the Weierstrass representation of $x$ has a pole of order two at $P$, a zero of order two at $Q$ and no other zeroes and poles.

So, $\omega / S$ is holomorphic, and then $b_{s}(Q)=1$.

This fact and (15) yield

$$
\left(c_{1}+d_{1}\right)\left(\left(c_{1}-d_{1}\right)^{2}-4\right)=0 .
$$

Taking into account (16) and after a suitable change of parameter

$$
\begin{aligned}
& \bar{M}=\left\{\left(z_{3}, w_{3}\right) \in(\mathbf{C} \cup\{\infty\})^{2} \mid w_{3}^{2}=\left(z_{3}^{2}-1\right)\left(z_{3}^{2}-c^{2}\right)\right\}, \\
& g=A_{2} z_{3}+B_{2}, \quad \omega=C_{1}\left(w_{3}+z_{3}^{2}-\frac{c^{2}+1}{2}\right) \frac{d z_{3}}{w_{3}},
\end{aligned}
$$

where $c, A_{2}, B_{2}, C_{1} \in \mathbf{C}, c \neq 1,-1,0, A_{2}, C_{1} \neq 0$.

Taking $z=w_{3}+z_{3}^{2}-\left(c^{2}+1\right) / 2$, we have $\operatorname{Degree}(x)=2$ and

$$
\begin{gathered}
\bar{M}=\left\{(z, w) \in(\mathbf{C} \cup\{\infty\})^{2} \mid w^{2}=(z-a)\left(z^{2}-1\right)\right\}, \\
g=A \frac{w}{z-a}+B, \quad \omega=C \frac{z-a}{w} d x,
\end{gathered}
$$

where $a, A, B, C \in \mathbf{C}, a \neq 1,-1,0, A, C \neq 0$.

As the 1-forms $\phi_{i}, i=1,2,3$, do not have real periods, (3) and the proof of Lemma 3 give

$$
\begin{aligned}
& \text { (1) } B C f_{i}(a) \in \mathbf{R}, \\
& \text { (2) } C f_{i}(a)=\bar{C} \bar{B}^{2} \bar{f}_{i}(a)+\bar{C} \bar{A}^{2} \bar{g}_{i}(a), \quad i=1,2 .
\end{aligned}
$$

If $B \neq 0$, from (1) and (2)

$$
C\left(1-|B|^{2}\right) f_{i}(a)=\frac{\bar{B} C A^{2}}{B} g_{i}(a), \quad i=1,2 .
$$

This fact contradicts Lemma 3. 
Therefore $B=0$, and looking at (17), $x$ is well defined if and only if

$$
\left(f_{1} \bar{g}_{2}-f_{2} \bar{g}_{1}\right)(a)=0 .
$$

Using Lemma $2, f_{i}(a), g_{i}(a) \neq 0$, if $a \in \mathbf{C}-\{1,-1\}, i=1,2$, and then (18) involves

$$
\log \left|\frac{f_{1} g_{2}}{f_{2} g_{1}}\right|(a)=0 .
$$

Consider $h: \Omega \rightarrow \mathbf{R}^{3}$ defined by

$$
h(a)=\log \left|\frac{f_{1} g_{2}}{f_{2} g_{1}}\right|(a) .
$$

From Lemma $2, h$ is a continuous function on $\Omega$, harmonic in $\stackrel{\circ}{\Omega}$. Using (4), $h(a)=0$ if $a \in i \mathbf{R} \cup\left\{\infty_{1}, \infty_{2}\right\}$, and if $h(a)=0$ then $h(\bar{a})=h(-a)=0$.

On the other hand, Lemma 4 shows that $h\left(a_{i}\right) \neq 0$, where $a_{i} \in l_{i}, a_{i} \neq \infty_{i}$, $i=1,2$.

Maximum principle for harmonic functions yields

$$
h(a)=0 \text { if and only if } a \in i \mathbf{R} \cup\left\{\infty_{1}, \infty_{2}\right\} .
$$

But if $a \in i \mathbf{R}$, Lemma 5 tells us that (18) holds if and only if $a=0$. This completes the proof of the theorem. Q.E.D.

The surface in Theorem 1 was discovered by Chen and Gackstatter, and we label it as a Chen-Gackstatter surface.

2.2. Second case. $b_{g}(P)=1$. As in the first case, we need some previous results.

Lemma 6. If $a_{i} \in l_{i}-\left\{1,-1, \infty_{i}\right\}, i=1,2$,

$$
\operatorname{Im}\left(3 g_{1} g_{2}-4 f_{1} f_{2}+2 a\left(g_{1} f_{2}+g_{2} f_{1}\right)\right)\left(a_{i}\right) \neq 0 .
$$

Proof. Define

$j\left(a_{i}\right)=\operatorname{Im}\left(3 g_{1} g_{2}-4 f_{1} f_{2}+2 a\left(g_{1} f_{2}+g_{2} f_{1}\right)\right)\left(a_{i}\right), \quad a_{i} \in l_{i}-\left\{\infty_{i}\right\}, \quad i=1,2$.

Using (4) and Remark 4, it is sufficient to prove

$$
j\left(a_{1}\right)=\left(3 \operatorname{Re}\left(g_{1}\right) g_{2}-4 \operatorname{Re}\left(f_{1}\right) f_{2}+2 a\left(\operatorname{Re}\left(g_{1}\right) f_{2}+\operatorname{Re}\left(f_{1}\right) g_{2}\right)\right)\left(a_{1}\right) \neq 0
$$

if $\left.a_{1} \in\right] 1,+\infty,\left[\subset l_{1}\right.$.

From Remark 5,

$$
j^{\prime}(a)=\frac{-2 a}{1-a^{2}}\left(j(a)+\frac{a^{2}-1}{a}\left(\operatorname{Re}\left(g_{1}\right) f_{2}+\operatorname{Re}\left(f_{1}\right) g_{2}\right)(a)\right)
$$

and looking at (7),

$$
\lim _{a_{1} \rightarrow 1}-i \frac{j\left(a_{1}\right)}{a_{1}-1}>0 .
$$

If $j$ vanishes at some point $\left.a_{1}^{\prime} \in\right] 1, \infty_{1}\left[\right.$, take $a_{0}$ the first such point, $a_{0}>$ 1. Remark 6 and (19) gives $-i j^{\prime}\left(a_{0}\right)>0$, and therefore $-i j\left(a_{1}\right)<0$, for each $\left.a_{1} \in\right] 1, a_{0}[$, which is contrary to (20). Q.E.D. 
Lemma 7. If $a \in i \mathbf{R},|a| \geq \sqrt{3}$,

$$
\operatorname{Re}\left(3 g_{1}^{2}(a)-4 f_{1}^{2}(a)+4 a f_{1}(a) g_{1}(a)\right) \neq 0 .
$$

Proof. Fixing the same notation as Lemma 5, if $i y \in i \mathbf{R}$, define

$$
\begin{aligned}
k(y) & =\operatorname{Re}\left(3 g_{1}^{2}(i y)-4 f_{1}^{2}(i y)+4 i y f_{1}(i y) g_{1}(i y)\right) \\
& =3\left(u_{1}^{2}(y)-v_{1}^{2}(y)\right)+4\left(s_{1}^{2}(y)-t_{1}^{2}(y)\right)-4 y\left(u_{1}(y) s_{1}(y)+t_{1}(y) v_{1}(y)\right)
\end{aligned}
$$

where $y \in \mathbf{R}$.

From (12),

$$
k^{\prime}(y)=\frac{2 y}{1+y^{2}}\left(k(y)-2 \frac{1+y^{2}}{y}\left(u_{1}(y) s_{1}(y)+t_{1}(y) v_{1}(y)\right)\right) .
$$

On the other hand, the function $s:]-\infty, 0] \rightarrow \mathbf{R}$ defined by

$$
s(a)=\left(\sqrt{3} g_{1}-2 f_{1}\right)(a)
$$

satisfy (see (7) and Remark 5)

$$
\begin{gathered}
s^{\prime}(a)=\frac{1}{1-a^{2}}\left((a+\sqrt{3}) f_{1}(a)-\frac{3}{2}(1+\sqrt{3} a) g_{1}(a)\right), \\
s^{\prime \prime}(a)=\frac{1}{2\left(a^{2}-1\right)} f_{1}(a)+\frac{3 \sqrt{3}}{4\left(a^{2}-1\right)} g_{1}(a), \quad s^{\prime}(-1)<0, s(-1)=0 .
\end{gathered}
$$

Thus, Remark 4 yields

$$
\left.s^{\prime \prime}(a)<0 \quad \text { for each } a \in\right]-1,1\left[, \quad s^{\prime}(0) s(0)<0,\right.
$$

and then, $s(0)<0$.

Thus

$$
k(0)=s(0)\left(\sqrt{3} g_{1}(0)+2 f_{1}(0)\right)>0 .
$$

Observe now

$$
k(\sqrt{3})=\left(\sqrt{3} g_{1}+2 i f_{1}\right)^{2}(i \sqrt{3}) .
$$

If we write

$$
H(x)=\frac{i x+1 / \sqrt{3}}{\sqrt{(x-i \sqrt{3})\left(x^{2}-1\right)}}
$$

from (3) and integrating by parts

$$
\left(\sqrt{3} g_{1}+2 i f_{1}\right)(i \sqrt{3})=4 \int_{-1}^{i \sqrt{3}} H(x) d x
$$

for a suitable choice of the above branch of $\sqrt{(x-i \sqrt{3})\left(x^{2}-1\right)}$.

If $x \in]-1,0[$, it is straightforward to check

$$
0<\operatorname{Re}(H)(x)<-\operatorname{Im}(H)(x) .
$$

After a suitable change of parameter

$$
\int_{0}^{i \sqrt{3}} H(x) d x=4(1+i) 3^{-1 / 4} \int_{-1}^{1 / 2} \frac{u}{\sqrt{u^{3}+1}} d u
$$


and therefore

$$
\operatorname{Re} \int_{0}^{i \sqrt{3}} H(x) d x=\operatorname{Im} \int_{0}^{i \sqrt{3}} H(x) d x<0 .
$$

So, (23) and (24) yield

$$
k(\sqrt{3})<0 .
$$

Remark 7 and (21) involve that $k$ vanishes only at one point $y_{0}>0$.

But $(25)$ and $(22)$ give $k(0) k(\sqrt{3})<0$. Therefore $\left.y_{0} \in\right] 0, \sqrt{3}[$ and $k(y) \neq$ $0, y \geq \sqrt{3}$. From (4), also $k(y) \neq 0, y \leq-\sqrt{3}$. Q.E.D.

Lemma 8. If $a \in]-\infty, 1[, a \neq-1$,

$$
\frac{3 g_{1}^{2}(a)-4 f_{1}^{2}(a)+4 a f_{1} g_{1}(a)}{1-a^{2}}>0 \text {. }
$$

Proof. By Remark 2, $k=3 g_{1}^{2}-4 f_{1}^{2}+4 a f_{1} g_{1}$ is well defined on ] $-\infty, 1[$.

Remark 5 gives

$$
k^{\prime}(a)=\frac{2 a}{a^{2}-1}\left(k(a)+\frac{2\left(a^{2}-1\right)}{a} f_{1}(a) g_{1}(a)\right) .
$$

Moreover, from (5) and (7)

$$
\left(\frac{k}{(a+1)^{2}}\right)(-1)=0, \quad\left(\frac{k}{(a+1)^{2}}\right)^{\prime}(-1)>0,
$$

and then

$$
k(-1-\varepsilon)<0, \quad k(-1+\varepsilon)>0,
$$

$\varepsilon>0$ small enough.

Suppose $k$ vanishes at a point $a^{\prime}<-1$, and take $a_{0}<-1$ the nearest point to -1 such that $k\left(a_{0}\right)=0$.

From (26), $k^{\prime}\left(a_{0}\right)>0$ and then $\left.k(a)>0, a \in\right] a_{0},-1[$, which is contrary to $(27)$.

So, $k(a)<0$ for each $a \in]-\infty,-1[$.

Analogously, $k(a)>0, a \in]-1,1[$, and the lemma holds. Q.E.D.

Lemma 9. For each $a \in \Omega-\left\{\infty_{1}, \infty_{2}, 1,-1\right\}$,

$$
\left(3 g_{1}^{2}(a)-4 f_{i}^{2}(a)+4 a f_{i}(a) g_{i}(a)\right) \neq 0 .
$$

Proof. Notice that from (4), we can suppose $i=1$.

Define now $\Omega_{+}^{\prime}=\{a \in \Omega \mid \operatorname{Im}(a)>0\}$ and $\Omega_{+}=\bar{\Omega}_{+}^{\prime}$.

Using again (4), we need only to prove the lemma for $a \in \Omega_{+}, a \neq \infty_{1}, 1$, -1 .

First, we will show that $\gamma: \partial \Omega_{+} \rightarrow \mathbf{C}$ defined by $\gamma(a)=f_{1}(a) / g_{1}(a)$ is a single closed curve.

For, we must have

$$
\gamma(b) \neq \gamma(c), \quad b, c \in \partial \Omega_{+}, \quad b \neq c .
$$

Note that $($ see $(8))$

$$
\lim _{|a| \rightarrow+\infty}\left|\frac{f_{1}}{g_{1}}\right|(a)=0
$$


Use Lemma 1 to obtain

$$
\left(\frac{f_{1}}{g_{1}}\right)^{\prime}=\frac{3 g_{1}^{2}-4 f_{1}^{2}+4 a g_{1} f_{1}}{4\left(1-a^{2}\right) g_{1}^{2}} .
$$

Hence, it follows from Remark 5 and Lemma 8 that $\gamma$ is injective on $[-\infty, 1]$.

Observe now that Remark 4 and Lemma 3 imply

$$
\operatorname{Im}\left(\frac{f_{1}}{g_{1}}\right)\left(a_{1}\right) \neq 0, \quad \text { for each } a_{1} \in\left[1,+\infty_{1}[\text {. }\right.
$$

Since $\gamma(a) \in \mathbf{R}, a \in[-\infty, 1]$, it remains only to check (28) for $b, c \in$ ] $1,+\infty_{1}[$.

If $\left.a_{1} \in\right] 1,+\infty{ }_{1}[$, Remark 4 yields

$$
\operatorname{Arg}\left(\frac{f_{1}}{g_{1}}\right)\left(a_{1}\right)=\operatorname{Arg}\left(\frac{\operatorname{Re}\left(f_{1}\right) \operatorname{Re}\left(g_{1}\right)-\left|f_{2}\right|\left|g_{2}\right|}{\operatorname{Re}\left(f_{1}\right)\left|g_{2}\right|+\operatorname{Re}\left(g_{1}\right)\left|f_{2}\right|}-i\right)\left(a_{1}\right)
$$

for each $\left.a_{1} \in\right] 1,+\infty_{1}[$.

Let $b$ denote the function

$$
\begin{gathered}
b:] 1,+\infty_{1}[\rightarrow \mathbf{R}, \\
b\left(a_{1}\right)=\frac{\operatorname{Re}\left(f_{1}\right) \operatorname{Re}\left(g_{1}\right)-\left|f_{2}\right|\left|g_{2}\right|}{\operatorname{Re}\left(f_{1}\right)\left|g_{2}\right|+\operatorname{Re}\left(g_{1}\right)\left|f_{2}\right|}\left(a_{1}\right) .
\end{gathered}
$$

By Lemma 3

$$
\left.\left(\operatorname{Re}\left(f_{1}\right)\left|g_{2}\right|+\operatorname{Re}\left(g_{1}\right)\left|f_{2}\right|\right)\left(a_{1}\right)=-\frac{4 \pi}{3}\left(a_{1}^{2}-1\right), \quad a_{1} \in\right] 1,+\infty_{1}[,
$$

and thus using Remarks 4 and 5

$$
b^{\prime}\left(a_{1}\right)=\frac{3}{4 \pi} \frac{(3 / 4)\left(\operatorname{Re}\left(g_{1}\right)^{2}+\left|g_{2}\right|^{2}\right)\left(a_{1}\right)+\left(\operatorname{Re}\left(f_{1}\right)^{2}+\left|f_{2}\right|^{2}\right)\left(a_{1}\right)}{\left(a_{1}^{2}-1\right)^{2}}>0 .
$$

Therefore $b$ is injective, then $\operatorname{Arg}\left(f_{1} / g_{1}\right)$ so is and (28) holds.

To conclude the lemma, notice that by $(29)$

$$
3 g_{1}^{2}(a)-4 f_{1}^{2}(a)+4 a g_{1}(a) f_{1}(a)=0 \text { if and only if }\left(\frac{f_{1}}{g_{1}}\right)^{\prime}(a)=0
$$

for each $a \in \Omega_{+}-\left\{\infty_{1}, 1,-1\right\}$.

Suppose $\left(f_{1} / g_{1}\right)^{\prime}\left(a_{0}\right)=0, a_{0} \in \stackrel{\circ}{\Omega}_{+}$and write $\alpha_{0}=\left(f_{1} / g_{1}\right)\left(a_{0}\right)$. Then

$$
2 \leq \frac{1}{2 \pi i} \int_{\partial \Omega_{+}} \frac{\left(f_{1} / g_{1}\right)^{\prime}(a)}{\left(f_{1} / g_{1}(a)-\alpha_{0}\right)} d a=n\left(\gamma\left(\partial \Omega_{+}\right), \alpha_{0}\right)
$$

where $n\left(\gamma\left(\partial \Omega_{+}\right), \alpha_{0}\right)$ is the winding number of $\gamma\left(\partial \Omega_{+}\right)$around $\alpha_{0}$.

But (28) involves $n\left(\gamma\left(\partial \Omega_{+}\right), f_{1}(a) / g_{1}\right)=1$ for each $a \in \stackrel{\circ}{\Omega}_{+}$, which is contrary to our assumption.

Taking into account Remark 4 and Lemmas 6 , 8, it is easy to obtain

$$
3 g_{1}^{2}(a)-4 f_{1}^{2}(a)+4 a f_{1}(a) g_{1}(a) \neq 0
$$

for $a \in \partial \Omega_{+}-\left\{\infty_{1}, 1,-1\right\}$.

This concludes the proof. Q.E.D. 


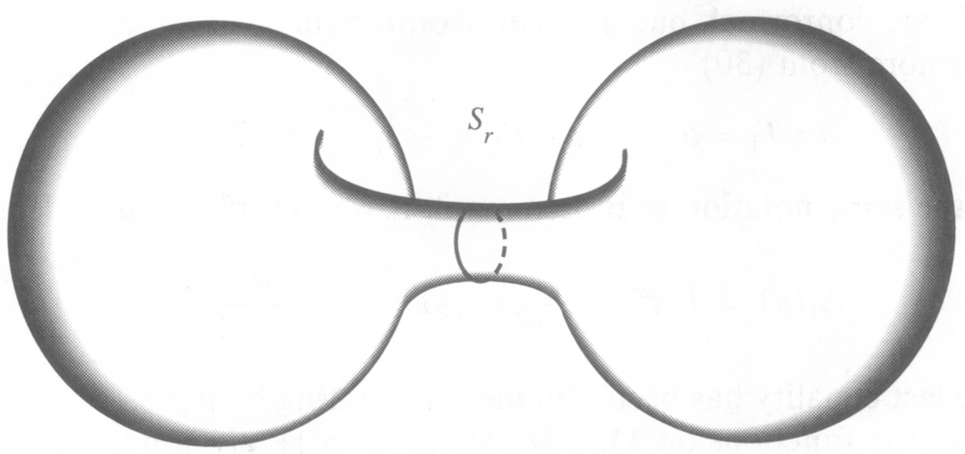

FIGURE 4

Take $r \in \mathbf{R}$ and consider the genus zero Riemann surface

$$
S_{r}=\left\{(\alpha, \beta) \in(\mathbf{C} \cup\{\infty\})^{2} \mid \alpha^{2}=-3 \beta^{2}+2 r \beta+3\right\} .
$$

In a way similar to that in Lemma 3 , a "concrete" representation of $S_{r}$ is given by cutting and joining two copies of the sphere.

In this case, we can cut each sheet along a smooth curve joining $\frac{r}{3}-\sqrt{1+\left(\frac{r}{3}\right)^{2}}$ and $\frac{r}{3}+\sqrt{1+\left(\frac{r}{3}\right)^{2}}$, for instance, the real interval

$$
\left[\frac{r}{3}-\sqrt{1+\left(\frac{r}{3}\right)^{2}}, \frac{r}{3}+\sqrt{1+\left(\frac{r}{3}\right)^{2}}\right] .
$$

See Figure 4.

Let $a$ denote the meromorphic function on $S_{r}$ defined by

$$
a: S_{r} \rightarrow \mathbf{C}, \quad a(\alpha, \beta)=\frac{r-3 \beta}{\alpha} .
$$

Observe that $\operatorname{Degree}(a)=2$ and denote by $\Omega_{r}$ the closure of $a^{-1}(\stackrel{\circ}{\Omega})$.

Concretely, cut $S_{r}$ along the two lines $l^{1}, l^{2}$ contained in $a^{-1}\{z \in \mathbf{R} \cup$ $\{\infty\}|| z \mid \geq 1\}$ :

$$
\begin{aligned}
& l^{1}=\left\{(\alpha, \beta) \in S_{r} \mid \beta \in\left[\frac{r}{3}+\frac{1}{2} \sqrt{1+\left(\frac{r}{3}\right)^{2}}, \frac{r}{3}+\sqrt{1+\left(\frac{r}{3}\right)^{2}}\right]\right\}, \\
& l^{2}=\left\{(\alpha, \beta) \in S_{r} \mid \beta \in\left[\frac{r}{3}-\sqrt{1+\left(\frac{r}{3}\right)^{2}}, \frac{r}{3}-\frac{1}{2} \sqrt{1+\left(\frac{r}{3}\right)^{2}}\right]\right\} .
\end{aligned}
$$

Then, open along $l^{1}, l^{2}$ and $\Omega_{r}$ is the closure of the resulting domain.

In $\Omega_{r}$ we have two copies of each line $l^{1}, l^{2}$. We will denote these copies by: $l_{j}^{1}, l_{j}^{2}, j=1,2$, respectively, and write $(\alpha, \beta)_{1}^{i},(\alpha, \beta)_{2}^{i}$ the two points corresponding to $(\alpha, \beta) \in l^{i}, i=1,2$.

Denote by $T_{i}, i=1,2,3$, the following automorphisms of $S_{r}$ :

$$
T_{1}(\alpha, \beta)=\left(-\alpha,-\beta+\frac{2 r}{3}\right), \quad T_{2}(\alpha, \beta)=(-\alpha, \beta), \quad T_{3}(\alpha, \beta)=(\bar{\alpha}, \bar{\beta})
$$

for each $(\alpha, \beta) \in S_{r}$. 
$T_{1}, T_{2}$ are conformal, but $T_{3}$ is anticonformal.

Furthermore from (30)

$$
a \circ T_{1}=a, \quad a \circ T_{2}=-a, \quad a \circ T_{3}=\bar{a} .
$$

Using the same notation as in Lemma 3 , if we put $\tau^{a}=\frac{1}{w} d z$, define

$$
\Delta_{1}(a)=\int_{\gamma_{i}} \tau^{a}=\frac{3}{2\left(a^{2}-1\right)} g_{i}(a)-\frac{a}{a^{2}-1} f_{i}(a)
$$

where the last equality has been obtained integrating by parts.

Consider the functions on $\Omega_{r}-\left\{a^{-1}\{1,-1, \infty\}\right\}$ given by

$$
\begin{aligned}
& \theta_{i}(\alpha, \beta)=\left(\left(-\sqrt{1+\left(\frac{r}{3}\right)^{2}}+2\left(\beta-\frac{r}{3}\right)\right) \Delta_{i}(a)-\alpha f_{i}(a)\right), \quad i=1,2, \\
& \sigma_{i}(\alpha, \beta)=\left(\left(\sqrt{1+\left(\frac{r}{3}\right)^{2}}+2\left(\beta-\frac{r}{3}\right)\right) \Delta_{i}(a)-\alpha f_{i}(a)\right), \quad i=1,2 .
\end{aligned}
$$

Let $B: \Omega_{r}-\left\{a^{-1}\{1,-1\}\right\} \rightarrow \mathbf{R}$ defined by

$$
B(\alpha, \beta)=\log \left|\frac{\theta_{1} \sigma_{2}}{\theta_{2} \sigma_{1}}\right|(\alpha, \beta) .
$$

Lemma 10. $B$ is a continuous function on $\Omega_{r}-\left\{a^{-1}\{1,-1\}\right\}$, harmonic on $\stackrel{\circ}{\Omega}_{r}$, verifying

$$
B \circ T_{1}=-B, \quad \begin{aligned}
B \circ T_{2} & =-B, \quad B \circ T_{3}=B, \\
\lim _{|a| \rightarrow 1}|B| & =+\infty .
\end{aligned}
$$

Proof. First, note that from (32):

$$
\theta_{1} \sigma_{1}(\alpha, \beta)=\frac{\alpha^{2}}{4\left(a^{2}-1\right)}\left(3 g_{i}^{2}(a)-4 f_{i}^{2}(a)+4 a f_{i}(a) g_{i}(a)\right), \quad i=1,2 .
$$

By Lemma 9 and (33), $B$ is well defined if $a(\alpha, \beta) \neq 1,-1, \infty$.

On the other hand, (30) gives

$$
\begin{gathered}
a^{-1}\{\infty\}=\left\{\left(0, \frac{r}{3} \pm \sqrt{1+\left(\frac{r}{3}\right)^{2}}\right)\right\}, \\
a^{-1}\{1,-1\}=\left\{\left(\frac{3}{2} \sqrt{1+\left(\frac{r}{3}\right)^{2}}, \frac{r}{3} \pm \frac{1}{2} \sqrt{1+\left(\frac{r}{3}\right)^{2}}\right),\right. \\
\left.\left(-\frac{3}{2} \sqrt{1+\left(\frac{r}{3}\right)^{2}}, \frac{r}{3} \pm \frac{1}{2} \sqrt{1+\left(\frac{r}{3}\right)^{2}}\right)\right\} .
\end{gathered}
$$

Taking into account (8), (32) and (33)

$$
\lim _{|a| \rightarrow \infty} B=1
$$

and $B$ is well defined if $a=\infty$. 
Define

$$
\begin{array}{ll}
\nu_{i}(\alpha, \beta)=\alpha g_{i}(a)-2\left(\beta-\frac{r}{3}+\sqrt{1+\left(\frac{r}{3}\right)^{2}}\right) f_{i}(a), & i=1,2, \\
\mu_{i}(\alpha, \beta)=\alpha g_{i}(a)-2\left(\beta-\frac{r}{3}-\sqrt{1+\left(\frac{r}{3}\right)^{2}}\right) f_{i}(a), \quad i=1,2 .
\end{array}
$$

Then, using (32) and (33)

$$
B=\log \left|\frac{\nu_{1} \mu_{2}}{\nu_{2} \mu_{1}}\right|
$$

and therefore by (7), (4) and (36), (35) holds.

To deduce (34), use (4) and (31).

It is straightforward that $\Delta B=0$. Q.E.D.

Theorem 2. There does not exist any orientable complete genus one minimal surface in $\mathbf{R}^{3}$, of finite total curvature $-8 \pi$ which Gauss map is singular at its unique end.

Proof. Suppose $x: M \rightarrow \mathbf{R}^{3}$ is such a surface.

As usual, $g, \omega$ will denote the Weierstrass representation of $x$.

We know $M$ is conformally equivalent to $\bar{M}-\{P\}$, where $\bar{M}$ is a compact genus one Riemann surface.

Since $b_{g}(P)=1$, there exist three points $P_{1}, P_{2}, P_{3}, P \neq P_{i}, i=1,2,3$, such that $b_{g}(P)=1$.

Denote $c_{i}=g\left(P_{i}\right), I=1,2,3$. It is clear that $c_{i} \neq c_{j}, i \neq j$.

Therefore

$$
\bar{M}=\left\{(z, w) \in(\mathbf{C} \cup\{\infty\})^{2} \mid w^{2}=\left(z-c_{1}\right)\left(z-c_{2}\right)\left(z-c_{3}\right)\right\}
$$

and $g=z, \omega=A \cdot \frac{1}{w} d z$ (see (2)).

Up to a rigid motion in $\mathbf{R}^{3}$, we will suppose $r=c_{1}+c_{2}+c_{3} \in \mathbf{R}$.

For $\gamma_{i}, i=1,2$, homology basis of $\bar{M}$, put

$$
d_{i}=\int_{\gamma_{i}} \frac{1}{w} d z, \quad e_{i}=\int_{\gamma_{i}} \frac{z}{w} d z, \quad i=1,2 .
$$

Integrating by parts

$$
\int_{\gamma_{i}} \frac{z^{2}}{w} d z=\frac{2 r}{3} e_{i}-\frac{s}{3} d_{i}, \quad i=1,2,
$$

where $s=c_{1} c_{2}+c_{1} c_{3}+c_{2} c_{3}$.

As $x$ is well defined, from (1) and (37):

$$
\bar{A} \bar{d}_{i}=A\left(\frac{2 r}{3} e_{i}-\frac{s}{r} d_{i}\right), \quad A e_{i} \in i \mathbf{R} .
$$

Since $\operatorname{Im}\left(d_{2} / d_{1}\right) \neq 0$ (see [11]), (38) yields $s=-3$, and (38) gets

$$
\bar{A} \bar{d}_{i}=A\left(\frac{2 r}{3} e_{i}+d_{i}\right), \quad A e_{i} \in i \mathbf{R} .
$$

Consider $y: M \rightarrow \mathbf{R}^{3}$ defined by

$$
y=\left(x_{3}-\frac{r}{3} x_{1}, \sqrt{1+\left(\frac{r}{3}\right)^{2}} x_{2}, x_{1}+\frac{r}{3} x_{3}\right) .
$$


It is clear that $y=R \circ H \circ x$, where $R$ is a rigid motion and $H$ is a homothety.

Furthermore, the Weierstrass representation $\left(\psi_{1}, \psi_{2}, \psi_{3}\right)$ of $y$ is given by (see (37) and (1)):

$$
\begin{aligned}
& \psi_{1}=A\left(1+\left(\frac{r}{3}\right)^{2}\right) \frac{z}{w} d z+d k_{1}, \\
& \psi_{2}=i A \sqrt{1+\left(\frac{r}{3}\right)^{2}}\left(\frac{1}{w}+\frac{r}{3} \frac{z}{w}\right) d z+d k_{2}, \\
& \psi_{3}=d k_{3},
\end{aligned}
$$

for suitable $k_{i}, i=1,2,3$, meromorphic functions on $\bar{M}$.

Thus, $y$ is well defined if and only if

$$
\begin{aligned}
\left(\bar{d}_{1}\right. & \left.+\left(\sqrt{1+\left(\frac{r}{3}\right)^{2}}+\frac{r}{3}\right) \bar{e}_{1}\right)\left(\bar{d}_{2}+\left(-\sqrt{1+\left(\frac{r}{3}\right)^{2}}+\frac{r}{3}\right) e_{2}\right) \\
& =\left(\bar{d}_{2}+\left(\sqrt{1+\left(\frac{r}{3}\right)^{2}}+\frac{r}{3}\right) \bar{e}_{2}\right)\left(d_{1}+\left(-\sqrt{1+\left(\frac{r}{3}\right)^{2}}+\frac{r}{3}\right) e_{1}\right) .
\end{aligned}
$$

Put $c_{0}=-c_{1} c_{2} c_{3}$ and take $\left(\alpha_{0}, \beta_{0}\right) \in S_{r}$ such that

$$
\alpha_{0}^{2}\left(r-3 \beta_{0}\right)=\beta_{0}^{3}-r \beta_{0}^{2}-3 \beta_{0}+c_{0}
$$

Then, consider the following change of parameter:

$$
x=\frac{1}{\alpha_{0}} z-\frac{\beta_{0}}{\alpha_{0}} .
$$

Hence, using (3), (30) and (41), (40) becomes

$$
\left(\theta_{1} \bar{\sigma}_{2}-\theta_{2} \bar{\sigma}_{1}\right)\left(\alpha_{0}, \beta_{0}\right)=0 \text {. }
$$

Note that $a_{0}=a\left(\alpha_{0}, \beta_{0}\right) \neq 1,-1, \infty$ because of $c_{i} \neq c_{j}, i \neq j$.

By (32) and (30)

$$
\theta_{i} \sigma_{i}\left(\alpha_{0}, \beta_{0}\right)=\frac{\alpha_{0}^{2}}{4\left(a_{0}^{2}-1\right)}\left(3 g_{i}^{2}\left(a_{0}\right)-4 f_{i}^{2}\left(a_{0}\right)+4 a_{0} f_{i}\left(a_{0}\right) g_{i}\left(a_{0}\right)\right), \quad i=1,2,
$$

and therefore Lemma 9 gives $\theta_{i}\left(\alpha_{0}, \beta_{0}\right), \sigma_{i}\left(\alpha_{0}, \beta_{0}\right) \neq 0, i=1,2$.

So, (42) involves $B\left(\alpha_{0}, \beta_{0}\right)=0$.

On the other hand, define

$$
\Gamma=\left\{(\alpha, \beta) \in \Omega_{r} \mid \beta \in(\mathbf{R}-] \frac{r}{3}-\sqrt{1+\left(\frac{r}{3}\right)^{2}}, \frac{r}{3}+\sqrt{1+\left(\frac{r}{3}\right)^{2}}[\cup\{\infty\})\right\} \text {. }
$$

We observe (see (30)) that $\Gamma=a^{-1}(\{z \in i \mathbf{R} \cup\{\infty\}|| a \mid \geq \sqrt{3}\})$. Using (34)

$$
B(\alpha, \beta)=0 \quad \text { if }(\alpha, \beta) \in \Gamma \text {. }
$$

We will show that $B$ vanishes only on $\Gamma$.

If $(\alpha, \beta) \in l_{j}^{i}, i=1,2, j=1,2$, and $\alpha \neq 0$, we have $\alpha, \beta \in \mathbf{R}$. Suppose $a(\alpha, \beta)>1$. Using Remark 4, (32) and Lemma 3, $B(\alpha, \beta)_{1}^{1}=0$ if and only if

$$
\begin{aligned}
& \left(3 g_{2} \operatorname{Re}\left(g_{1}\right)-4 f_{2} \operatorname{Re}\left(f_{1}\right)\right)\left(a_{1}\right) \\
& \quad-2 a_{1}\left(\operatorname{Re}\left(g_{1}\right) f_{2}+\operatorname{Re}\left(f_{1}\right) g_{2}\right)\left(a_{1}\right)=0 .
\end{aligned}
$$




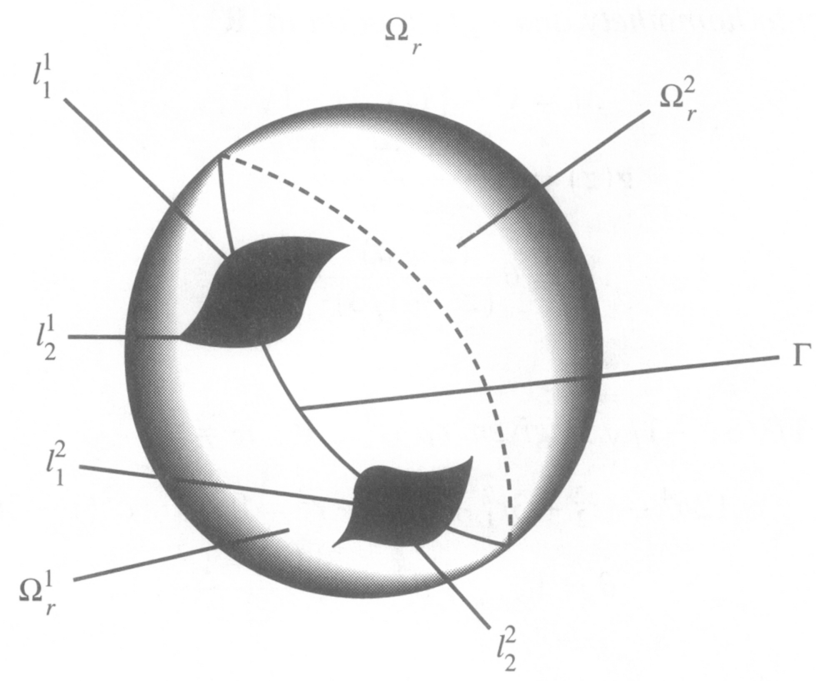

FIGURE 5

So, (34) and Lemma 6 get $B(\alpha, \beta) \neq 0,(\alpha, \beta) \in l_{j}^{i}, i=1,2, j=1,2$, $\alpha \neq 0$.

Let $\Omega_{r}^{1}, \Omega_{r}^{2}$ denote the two components of $\Omega_{r}-\Gamma$, and note that $\partial \Omega_{r}^{j} \subset$ $\Gamma \cup l_{1}^{1} \cup l_{1}^{2} \cup l_{2}^{1} \cup l_{2}^{2}$ (see Figure 5).

If $B(\alpha, \beta)=0,(\alpha, \beta) \notin \Gamma$, we have seen $(\alpha, \beta) \notin l_{j}^{i}, i=1,2, j=1,2$, and therefore $(\alpha, \beta)$ is an interior point of $\Omega_{r}^{1}$ or $\Omega_{r}^{2}$.

The symmetries in (34), together with the Maximum Principle for harmonic functions yields a contradiction.

So, if $(\alpha, \beta) \in \Omega_{r}$,

$$
B(\alpha, \beta)=0 \quad \text { if and only if } \quad(\alpha, \beta) \in \Gamma .
$$

Thus $\left(\alpha_{0}, \beta_{0}\right) \in \Gamma$, and obviously $\alpha_{0} \neq 0$.

Since $\alpha_{0} \in i \mathbf{R}, \beta_{0} \in \mathbf{R}$, then $a_{0} \in i \mathbf{R}$, and using (4), (32) and (42) we get

$$
\operatorname{Re}\left(3 f_{1}^{2}\left(a_{0}\right)-4 g_{1}^{2}\left(a_{0}\right)+4 a_{0} f_{1}\left(a_{0}\right) g_{1}\left(a_{0}\right)\right)=0
$$

which is contrary to Lemma 7.

This fact completes the proof. Q.E.D.

\section{GenUS Zero Minimal SURFACES OF TOTAL CURVATURE $-8 \pi$}

In the following, we will suppose $\gamma=\operatorname{Genus}(M)=0$.

Looking at Table 1, we can distinguish three different cases: $k=1, k=2$ and $k=3$.

First case. $k=3$. Jorge-Meeks formula (2) yields $I_{1}=I_{2}=I_{3}=1$.

After a suitable change of parameter in $\bar{M}=\mathbf{C} \cup\{\infty\}$, suppose that $1 / \sqrt{3}$, $-1 / \sqrt{3}$ and $\infty$ are the three ends of $M$, and up to rigid motion in $\mathbf{R}^{3}, g(\infty)=$ $\infty, b_{g}(\infty)=0$. 
Theorem 3. Up to homothety and rigid motion in $\mathbf{R}^{3}$ :

$$
\begin{aligned}
M & =\mathbf{C}-\{1 / \sqrt{3},-1 \sqrt{3}\}, \\
g(z) & =B \frac{z^{2}+c z+d}{z+a}, \\
\omega & =\theta \frac{(z+a)^{2}}{\left(z^{2}-1 / 3\right)^{2}} d z
\end{aligned}
$$

where

(1) if $a \neq 1 / \sqrt{3},-1 / \sqrt{3}$, given $r_{1}, r_{2} \in \mathbf{R}, r_{2} \neq 0$,

$$
\begin{gathered}
c=0, \quad 12 a^{4}-\left(r_{2}^{2}+3 r_{1}^{2}+4\right) a^{2}-r_{1}^{2}=0, \quad a^{2}(1-3 d)^{2}=r_{1}^{2}, \\
\theta=1, \quad B^{2}=\frac{3\left|3 a^{2}-1\right|^{2}}{r_{2}^{2}},
\end{gathered}
$$

$$
c=0, \quad a=1 / \sqrt{3},-1 / \sqrt{3}, \quad d=1, \quad \theta=1, \quad B \in \mathbf{R}-\{0\},
$$

Proof. Up to rigid motion and homothety, we can assume (45), where $B \in$ $\mathbf{R}-\{0\}$ and $|\theta|=1$.

Looking at (1), since $x$ is well defined (that is, $\phi_{k}, k=1,2,3$, do not have real periods):

$$
\begin{gathered}
c=0, \quad 3 \bar{a}^{2}-1=\theta^{2} B^{2}(1+3 d)(1-d), \\
\operatorname{Im}(\theta)=0, \quad \operatorname{Im}(\theta a(1-3 d))=0 .
\end{gathered}
$$

Thus, we can suppose $\theta=1$.

If $a \neq 1 / \sqrt{3},-1 / \sqrt{3}$, then $B^{2}=\left(3 \bar{a}^{2}-1\right) /((1+3 d)(1-d))$.

Writing $r_{1}=a(1-3 d)$ and $r_{2}^{2}=3\left(3 a^{2}-1\right)(1+3 d)(1-d)$, (46) holds.

If $a=1 / \sqrt{3},-1 / \sqrt{3},(48)$ gives $d=1$ or $d=-1 / 3$.

Since Degree $(g)=2, d \neq-1 / 3$ (see (45)). By (48), it is easy now to conclude the lemma. Q.E.D.

Definition 1. Denote by $\mathscr{F}$ the family of surfaces given by (45)-(46) and (47) satisfying $(3 d-1)^{2} \neq 12 a^{2}$.

Geometrically, $\mathscr{F}$ is the family of genus zero orientable complete minimal surfaces of finite total curvature $-8 \pi$ and three catenoid ends. Here, a catenoid end means an embedded end asymptotic to a catenoid. An embedded end $P_{i}$ is a catenoid end when the Gauss map $g$ is regular at $P_{i}$ (see [5]).

The Jorge-Meeks surface of degree 3 (the trinoid) is the first interesting example in $\mathscr{F}$.

3.2. Second case. $k=2$. We will suppose $M=\mathbf{C}-\{0\}$, that this, $P_{1}=\infty$ and $P_{2}=0$ are the two ends of $M$. Moreover, we can assume $g(\infty)=\infty$.

By formula (2) again, we have two possibilities: $I_{1}=I_{2}=2$ or $I_{1}=1$, $I_{2}=3$.

The following theorems are consequences of similar arguments that are given in Theorem 3. 
Theorem 4. Suppose $I_{1}=I_{2}=2$. Then, up to change of parameter in $\mathbf{C} \cup\{\infty\}$, homothety and rigid motion in $\mathbf{R}^{3}$ :

(1) If $g$ has a regular end (without loss of generality, assume $\infty$ is such an end $), g(0) \neq \infty$,

$$
M=\mathbf{C}-\{0\}, \quad g(z)=B \frac{z^{2}+c z+d}{z+1}, \quad \omega=\theta \frac{(z+1)^{2}}{z^{3}} d z,
$$

where

$|\theta|=1, \quad B \in \mathbf{R}-\{0\}, \quad-1=\theta^{2} B^{2}\left(c^{2}+2 d\right), \quad \theta(1+c) \in \mathbf{R}, \quad 1-c+d \neq 0$, and if $b_{g}(\infty)=0, g(0)=\infty$,

$$
M=\mathbf{C}-\{0\}, \quad g(z)=B \frac{z^{2}+c z+1}{z}, \quad \omega=\theta \frac{1}{z} d z,
$$

where

$$
\theta=i, \quad B \in \mathbf{R}-\{0\}, \quad-1=B^{2}\left(c^{2}+2\right) .
$$

(2) If $b_{g}(\infty)=b_{g}(0)=1$,

$$
M=\mathbf{C}-\{0\}, \quad g(z)=B z^{2}, \quad \omega=\theta \frac{1}{z^{3}} d z,
$$

where $B \in \mathbf{R}-\{0\}, \theta=1$.

Theorem 5. Suppose $I_{1}=1$ and $I_{2}=3$. Then, up to change of parameter in $\mathbf{C} \cup\{\infty\}$, homothety and rigid motion in $\mathbf{R}^{3}$ :

(1) If $b_{g}(\infty)=0, g(0) \neq \infty$,

$$
M=\mathbf{C}-\{0\}, \quad g(z)=B \frac{z^{2}+d}{z+1}, \quad \omega=\theta \frac{(z+1)^{2}}{z^{4}} d z
$$

where $B \in \mathbf{R}-\{0\}, \theta=1, d \in \mathbf{C}-\{-1\}$, and if $b_{g}(\infty)=0, g(0)=\infty$,

$$
M=\mathbf{C}-\{0\}, \quad g(z)=B \frac{z^{2}+1}{z}, \quad \omega=\theta \frac{1}{z^{2}} d z
$$

where $B \in \mathbf{R}-\{0\}, \theta=1$.

(2) If $b_{g}(\infty)=1, g(0) \neq 0$,

$$
M=\mathbf{C}-\{0\}, \quad g(z)=B\left(z^{2}+1\right), \quad \omega=\theta \frac{1}{z^{4}} d z
$$

where $B \in \mathbf{R}-\{0\}, \theta=1$ and if $b_{g}(\infty)=1, g(0)=0$,

$$
M=\mathbf{C}-\{0\}, \quad g(z)=B z^{2}, \quad \omega=\theta \frac{1}{z^{4}} d z
$$

where $B \in \mathbf{R}-\{0\}, \theta=1$.

3.3. Third case. $k=1$. In this case, (2) involves $I_{1}=5$.

Assume that $\infty$ is the unique end of $M$, and up to rotation in $\mathbf{R}^{3}, g(\infty)=$ $\infty$. As before, the following theorem holds. 
Theorem 6. Up to change of parameter in $\mathbf{C} \cup\{\infty\}$, homothety and rigid motion in $\mathbf{R}^{3}$ :

(1) If $b_{g}(\infty)=0$,

$$
M=\mathbf{C}, \quad g(z)=B \frac{z^{2}+c z+1}{z}, \quad \omega=\theta z^{2} d z
$$

where

(2) If $b_{g}(\infty)=1$,

$$
B \in \mathbf{R}-\{0\}, \quad c, \theta \in \mathbf{C}, \quad|\theta|=1 .
$$

$$
M=\mathbf{C}, \quad g(z)=B\left(z^{2}+c\right), \quad \omega=\theta d z
$$

where

$$
B \in \mathbf{R}-\{0\}, \quad c, \theta \in \mathbf{C}, \quad|\theta|=1 .
$$

\section{STATEMENT OF RESUlt}

An Osserman classical result (see [9]) classifies the catenoid and Enneper surface as the unique complete minimal surfaces with total curvature $-4 \pi$.

Thus, Theorems 1, 2, 3, 4, 5, and 6 imply our main result:

Corollary 1. Let $M$ be an orientable complete minimal surface in $\mathbf{R}^{3}$ of finite total curvature greater than $-12 \pi$.

Then, $M$ is one of the following surfaces: a plane, a catenoid, Enneper surface, a surface described in Theorems 3, 4, 5, 6, or Chen-Gackstatter surface.

\section{A GEOMETRIC CONSEQUENCE}

Finally, we prove the following fact, which is related to Osserman-Mo theorem in [7].

Corollary 2. Let $M$ be a orientable complete minimal surface in $\mathbf{R}^{3}$.

If the Gauss map $g$ takes on five distinct values (without counting multiplicities) at most once, then $M$ is one of the following surfaces: the plane, the catenoid, Enneper surface, a surface in the family $\mathscr{F}$ or Chen-Gackstatter surface.

Proof. First, by Osserman-Mo theorem in [7], $M$ has finite total curvature.

Since $M=\bar{M}-\left\{P_{1}, \ldots, P_{k}\right\}$ where $\bar{M}$ is a compact Riemann surface, and $g$ extends meromorphically to $\bar{M}$, we can define the total branching number $V$ of $g$ by

$$
V=\sum_{P \in \bar{M}} b_{g}(P) .
$$

It is well known (see [11]) that

$$
V=2 n+2 \gamma-2
$$

where, as usual, $\gamma=\operatorname{Genus}(\bar{M})$ and $n=\operatorname{Degree}(g)$.

Write by $b_{i}, i=1, \ldots, 5$, the five points in $\mathbf{S}^{2}$ such that

$$
\operatorname{Card}\left(\left(g^{-1}\left\{b_{i}\right\}\right) \cap M\right) \leq 1,
$$

$i=1, \ldots, 5$, where "Card" means cardinality. 
By (53),

$$
k+5 \geq \operatorname{Card}\left(g^{-1}\left\{b_{1}, \ldots, b_{5}\right\}\right) \geq 5 n-V .
$$

Then (54) and (2) yield

$$
2 k+\gamma \leq 6 .
$$

If $\gamma \geq 5$, (55) gives $k=0$, a contradiction. Assume $\gamma=3,4$. In this case, (55) involves $k=1$. Looking at (53), we deduce

$$
V \geq(n-2)+4(n-1) \text {, }
$$

that is $($ see $(52))$,

$$
2 n \leq 2 \gamma+4
$$

Hence, using (2) and (56), we conclude $I_{1}=1$, a contradiction.

In a similar way, if $\gamma=2$, then $k=2$ and $I_{1}=I_{2}=1$ or $k=1$ and $I_{1}=1$. Schoen (see [10]) characterized first surfaces as catenoids and pairs of planes. So, this case is also impossible.

Suppose now $\gamma=1$.

From (54) and (2), $k=2$ and $I_{1}=I_{2}=1$ or $k=1$ and $I_{1}=3$.

As before, the first case gets a contradiction. Taking into account Theorems 1 and 2 and (2), the second case corresponds to Chen-Gackstatter surface.

Finally, $\gamma=0$ involves (see (54) and (2)):

(1) $k=1$ and $I_{1}=3$, which gives Enneper's surface (see [9]).

(2) $k=2$ and $I_{1}=I_{2}=1$, that is, a catenoid (see [9]).

(3) $k=3$ and $I_{1}=I_{2}=I_{3}=1$. Observe that (2) yields $n=2$, and (53) implies $b_{g}\left(P_{i}\right)=0, i=1,2,3$. These are the surfaces in $\mathscr{F}$.

This fact completes the proof. Q.E.D.

Note added in proof. The author has learned that D. Bloss has obtained Theorems 1 and 2 using different techniques (D. Bloss, Elliptische Funktionen und Vollständige Minimalflächen, Ph.D. thesis, Freien Universität Berlin, Berlin, November, 1989.)

\section{REFERENCES}

1. C. C. Chen and F. Gackstatter, Elliptic and hyperelliptic functions and complete minimal surfaces with handles, IME-USP 27 (1981).

2. C. J. Costa, Classification of complete minimal surfaces in $\mathbf{R}^{3}$ with total curvature $12 \pi$, (preprint).

3. D. Hoffman and W. H. Meeks III, One parameter families of embedded minimal surfaces, (in preparation).

4. __, A complete embedded minimal surface in $\mathbf{R}^{3}$ with genus one and three ends, J. Differential Geom. 21 (1985).

5. L. P. Jorge and W. H. Meeks III, The topology of complete minimal surfaces of finite total Gaussian curvature, Topology 22 (1983).

6. W. H. Meeks III, The classification of complete minimal surfaces in $\mathbf{R}^{3}$ with total curvature greater than $-8 \pi$, Duke Math. J. 48 (1981).

7. X. Mo and R. Osserman, On the Gauss map and total curvature of complete minimal surfaces and extension of Fujimoto's theorem, (preprint).

8. R. Osserman, Global properties of minimal surfaces in $E^{3}$ and $E^{n}$, Ann. of Math. (2) 80 (1964). 
9. __, A survey of minimal surfaces, (2nd ed.), Dover, New York, 1986.

10. R. N. Schoen, Uniqueness, symmetry and embeddedness of minimal surfaces, J. Differential Geom. 18 (1983).

11. G. Springer, Introduction to Riemann surfaces, Addison-Wesley, 1957.

12. E. L. Barbanel, Complete minimal surfaces in $\mathbf{R}^{3}$ of low total curvature, Thesis, Univ. of Massachusetts, 1987.

13. Yi Fang, Complete minimal surfaces of finite total curvature, Thesis, Univ. of Massachusetts, 1990.

Departamento de Geometria y Topologia, Facultad de Ciencias, Universidad de Granada, 18071 Granada, Spain

E-mail address: fjlopez@ugr.es 